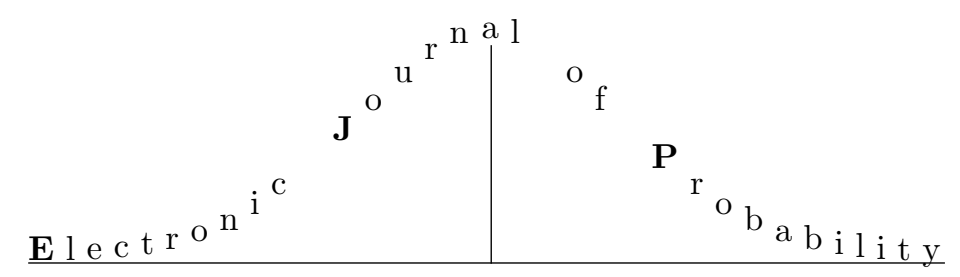

Vol. 11 (2006), Paper no. 22, pages 540-562.

Journal URL

http://www.math.washington.edu/〜ejpecp/

\title{
Examples of Condition $(T)$ for Diffusions in a Random Environment
}

\author{
Tom Schmitz \\ Department of Mathematics \\ ETH Zurich \\ CH-8092 Zurich \\ Switzerland \\ email: schmitz@math.ethz.ch
}

\begin{abstract}
With the help of the methods developed in [21], we highlight condition $(T)$ as a source of new examples of 'ballistic' diffusions in a random environment when $d \geq 2$ ('ballistic' means that a strong law of large numbers with non-vanishing limiting velocity holds). In particular we are able to treat the case of non-constant diffusion coefficients, a feature that causes problems. Further we recover the ballistic character of two important classes of diffusions in a random environment by simply checking condition $(T)$. This not only points out to the broad range of examples where condition $(T)$ can be checked, but also fortifies our belief that condition $(T)$ is a natural contender for the characterisation of ballistic diffusions in a random environment when $d \geq 2$
\end{abstract}

Key words: Diffusions in a random environment, ballistic behavior, Condition $(T)$

AMS 2000 Subject Classification: Primary 60K37, 82D30.

Submitted to EJP on October 25 2005, final version accepted July 102006. 


\section{Introduction}

Diffusions in random environment in higher dimensions are still poorly understood. An important tool in the study of diffusions in random environment has been the "method of the environment viewed from the particle", cf. [16], [17] and the references therein, with the serious drawback that it only applies in very specific situations where one can find an (most often explicit) invariant measure for the process of the environment viewed from the particle. However new ideas recently emerged in the discrete framework of random walks in random environment that apply in the general setting, see for instance [30], [33] and the references therein. In particular the renewal-type arguments introduced in Sznitman-Zerner [31], and related developments centered around condition ( $T$ ) in Sznitman [27], [28], [29] shed some light on walks with non-vanishing limiting velocity. Let us as well point out the recent breakthrough in the diffusive regime, cf. Sznitman and Zeitouni [32], where new methods falling outside the framework of these renewal techniques are developed.

In Shen [22] a renewal structure in the spirit of [31] was implemented in the continuous space-time setting. In our recent work [21], we built up on these results, and showed that condition $(T)$ is also instrumental in the continuous setting: when $d \geq 2$, it implies a strong law of large numbers with non-vanishing limiting velocity (which we refer to as ballistic behavior) and an invariance principle governing corrections to the law of large numbers.

This article mainly follows two objectives. We first highlight condition $(T)$ as a source of new examples of ballistic diffusions in random environment when $d \geq 2$. Second, we rederive the well-known ballistic character of a class of diffusions in random environment with a divergence-free drift field by simply checking condition $(T)$. We also check condition $(T)$ for an anisotropic gradient-type diffusion, the ballistic nature of which was shown in Shen [22]. This not only points out to the broad range of examples where condition $(T)$ can be checked, but also fortifies our belief that condition $(T)$ is a natural contender for the characterisation of ballistic behavior.

Before describing our results in more details, let us recall the setting.

The random environment $\omega$ is described by a probability space $(\Omega, \mathcal{A}, \mathbb{P})$. We assume that there exists a group $\left\{t_{x}: x \in \mathbb{R}^{d}\right\}$ of transformations on $\Omega$, jointly measurable in $x, \omega$, which preserve the probability $\mathbb{P}$ :

$$
t_{x} \mathbb{P}=\mathbb{P} .
$$

On $(\Omega, \mathcal{A}, \mathbb{P})$ we consider random variables $a(\cdot)$ and $b(\cdot)$ with respective values in the space of symmetric $d \times d$ matrices and $\mathbb{R}^{d}$, and we define

$$
a(x, \omega) \stackrel{\text { def }}{=} a\left(t_{x} \omega\right), \quad b(x, \omega) \stackrel{\text { def }}{=} b\left(t_{x} \omega\right) .
$$

We shall assume that $a(\cdot, \omega)$ is uniformly elliptic and bounded, and that $b(\cdot, \omega)$ is bounded, i.e. there are constants $\nu \geq 1, \beta>0$, such that for all $\omega \in \Omega$, all $x, y \in \mathbb{R}^{d}$,

$$
\frac{1}{\nu}|y|^{2} \leq y^{t} \cdot a(x, \omega) y \leq \nu|y|^{2}, \quad|b(x, \omega)| \leq \beta,
$$

where $|\cdot|$ denotes the Euclidean norm for vectors resp. for square matrices, and $y^{t}$ stands for the transposed vector of $y$. We assume as well that $a(\cdot, \omega), b(\cdot, \omega)$ are Lipschitz continuous, i.e. there is a constant $K>0$ such that for all $\omega \in \Omega, x, y \in \mathbb{R}^{d}$,

$$
|a(x, \omega)-a(y, \omega)|+|b(x, \omega)-b(y, \omega)| \leq K|x-y| .
$$


We will further assume finite range dependence, i.e. there is an $R>0$ such that, when we define the $\sigma$-field $\mathcal{H}_{F} \stackrel{\text { def }}{=} \sigma\{a(x, \cdot), b(x, \cdot): x \in F\}, F$ a Borel subset of $\mathbb{R}^{d}$, then

$$
\mathcal{H}_{A} \text { and } \mathcal{H}_{B} \text { are } \mathbb{P} \text {-independent when } d(A, B)>R,
$$

where $d(A, B)=\inf \{|x-y|: x \in A, y \in B\}$. We define the differential operator attached to $a(\cdot, \omega), b(\cdot, \omega)$,

$$
\mathcal{L}_{\omega}=\frac{1}{2} \sum_{i, j=1}^{d} a_{i j}(x, \omega) \partial_{i j}^{2}+\sum_{i=1}^{d} b_{i}(x, \omega) \partial_{i},
$$

and, for $\omega \in \Omega$, we denote with $P_{x, \omega}$ the unique solution to the martingale problem for $\mathcal{L}_{\omega}$ started at $x \in \mathbb{R}^{d}$, see Theorem 4.3 p.146 in [1]. The laws $P_{x, \omega}$ describe the diffusion in the environment $\omega$. We write $E_{x, \omega}$ for the corresponding expectation. We denote with $\left(X_{t}\right)_{t \geq 0}$ the canonical process on $C\left(\mathbb{R}_{+}, \mathbb{R}^{d}\right)$, with $\left(\mathcal{F}_{t}\right)_{t \geq 0}$ the canonical filtration, and $\mathcal{F}$ the Borel $\sigma$-field on $C\left(\mathbb{R}_{+}, \mathbb{R}^{d}\right)$. The laws $P_{x, \omega}$ are usually called the quenched laws. To restore some stationarity to the problem, it is convenient to introduce the annealed laws $P_{x}$, which are defined as the semi-direct products:

$$
P_{x} \stackrel{\text { def }}{=} \mathbb{P} \times P_{x, \omega},, \text { for } x \in \mathbb{R}^{d} .
$$

The corresponding expectations are denoted with $E_{x}=\mathbb{E} \times E_{x, \omega}$, where $\mathbb{E}$ denotes the expectation with respect to the measure $\mathbb{P}$. Observe that the Markov property is typically lost under the annealed laws.

Let us now explain in more details the purpose of this work. We first recall from [21] the definition of condition $(T)$ (where $T$ stands for transience). Introduce, for $\ell$ a unit vector of $\mathbb{R}^{d}$, $b, L>0$, the slabs $U_{\ell, b, L} \stackrel{\text { def }}{=}\left\{x \in \mathbb{R}^{d}:-b L<x \cdot \ell<L\right\}$.

Definition 1.1. We say that condition $(T)$ holds relative to $\ell \in S^{d-1}$, in shorthand notation $(T) \mid \ell$, if for all $\ell^{\prime} \in S^{d-1}$ in a neighborhood of $\ell$, and for all $b>0$,

$$
\limsup _{L \rightarrow \infty} L^{-1} \log P_{0}\left[X_{T_{U_{\ell^{\prime}, b, L}}} \cdot \ell^{\prime}<0\right]<0,
$$

where $T_{U_{\ell^{\prime}, b, L}}$ denotes the exit time from the slab $U_{\ell^{\prime}, b, L}$.

Let us recall that, when $d \geq 2$, and under our standing assumptions (1)-(5), condition $(T)$ implies a ballistic strong law of large numbers and an annealed invariance principle governing corrections to the law of large numbers, see [21]:

$$
P_{0}-\text { a.s., } \quad \frac{X_{t}}{t} \rightarrow \tilde{v}, \quad \tilde{v} \neq 0, \text { deterministic, with } \tilde{v} \cdot \ell>0,
$$

and under $P_{0}, B^{s}=\frac{X \cdot s-\cdot s \tilde{v}}{\sqrt{s}}$ converges in law on $C\left(\mathbb{R}_{+}, \mathbb{R}^{d}\right)$, as $s \rightarrow \infty$, to a

Brownian motion $B$. with non-degenerate covariance matrix.

In Section 2, we provide a new class of examples where condition $(T)$ holds. Namely, we prove that there is a constant $c_{1}(d, \nu, \beta, K, R)>1$ such that, for $\ell \in S^{d-1}$, the inequality

$$
\mathbb{E}\left[(b(0, \omega) \cdot \ell)_{+}\right]>c_{1} \mathbb{E}\left[(b(0, \omega) \cdot \ell)_{-}\right]
$$


implies condition $(T) \mid \ell$, see Theorem 2.1. This theorem extends the result of Theorem 5.2 in [21] in the sense that we drop the assumption that the diffusion coefficient $a(\cdot, \omega)$ equals the identity for all $\omega \in \Omega$. This is more than a technical improvement, for this new set-up leads to severe difficulties. Let us recall that in [21] we introduced for each bounded $C^{\infty}$-domain $U$ containing 0 , an auxiliary diffusion with characteristics independent of the environment that exhibited the same exit distribution from $U$ as the annealed diffusion in the random environment when starting at 0 . With the objective of checking condition $(T)$, we approximated the slab $U_{\ell, b, L}$ by bounded $C^{\infty}$-domains, and using the auxiliary diffusion, we were able to restore some Markovian character to the question of controlling the exit distribution from the slab $U_{\ell, b, L}$ under the annealed measure $P_{0}$. We keep this strategy in the present article, with the difference that now, when $d \geq 2$, the construction of an auxiliary diffusion exhibiting the same properties as the auxiliary diffusion from [21] described above is more intricate.

Inspired by [21], we are naturally led to choose for a bounded $C^{\infty}$ - domain $U$ containing 0 the following auxiliary diffusion and drift coefficients:

$$
\begin{array}{ll}
a_{U}^{\prime}(x)=\frac{\mathbb{E}\left[g_{\omega, U}(x) a(x, \omega)\right]}{\mathbb{E}\left[g_{\omega, U}(x)\right]}, \text { if } x \in U \backslash\{0\}, \quad a_{U}^{\prime}(x)=I d, \text { else, } \\
b_{U}^{\prime}(x)=\frac{\mathbb{E}\left[g_{\omega, U}(x) b(x, \omega)\right]}{\mathbb{E}\left[g_{\omega, U}(x)\right]}, \text { if } x \in U \backslash\{0\}, \quad b_{U}^{\prime}(x)=0, \quad \text { else, }
\end{array}
$$

where $g_{\omega, U}$ is the Green function corresponding to the quenched diffusion started at 0 and killed when exiting $U$. Notice that, when $a(\cdot, \omega) \equiv I d$ for all $\omega \in \Omega$ as in [21], then $a_{U}^{\prime} \equiv I d$, and we recover the auxiliary diffusion from [21], i.e. a Brownian motion perturbed by a bounded measurable drift $b_{U}^{\prime}$. In the present setting, $a_{U}^{\prime}$ is uniformly elliptic and bounded, but in general discontinuous at 0 and at the boundary $\partial U$. The massive discontinuity at the boundary may very well imply nonuniqueness for the martingale problem attached to $a_{U}^{\prime}$ and $b_{U}^{\prime}$.

Thus, in the key Proposition 2.4, we prove the existence of a solution $P_{0, U}^{\prime}$ to the martingale problem for $\mathcal{L}^{\prime}$ started at 0 such that the respective exit distributions from $U$ under $P_{0, U}^{\prime}$ and the annealed measure $P_{0}$ agree. Any such measure will serve as an auxiliary measure.

We cannot adapt the methods used in [21] to prove Proposition 2.4 in the present setting. Due to the discontinuity of $a_{U}^{\prime}$ at 0 , a certain Dirichlet problem in the domain $U$, attached to the auxiliary coefficients $a_{U}^{\prime}, b_{U}^{\prime}$, may fail to have a strong solution in the Sobolev space $W^{2, p}(U)$, $p \geq 1$. This is in contrast to the situation in [21], where $a_{U}^{\prime} \equiv I d$ implied the existence of a solution with good differentiability properties to the above Dirichlet problem. As a result, when choosing arbitrary smooth and bounded approximations $b_{n}$ of the auxiliary drift $b_{U}^{\prime}$, we obtained convergence of the smooth solutions, together with their derivatives, of the perturbed Dirichlet problems attached to $a_{n} \equiv I d$ and $b_{n}$. This enabled us to conclude the proof of the result corresponding to Proposition 2.4 in the setting of [21], see also Remark 2.7.

To avoid controls on derivatives in the present setting, we use results of Krylov [10], [11]. The specific choice of the auxiliary coefficients $a_{U}^{\prime}, b_{U}^{\prime}$ enables us to construct a diffusion started at 0 and attached to $a_{U}^{\prime}, b_{U}^{\prime}$, that, killed when exiting $U$, admits $\mathbb{E}\left[g_{\omega, U}\right]$ as occupation time density in $U$. This implies that the above diffusion exhibits the same exit distribution from $U$ as the annealed diffusion in random environment.

In Section 3, we study a Brownian motion perturbed by a random divergence-free drift field the $\mathbb{P}$-expectation of which does not vanish. The ballistic character of this class of diffusions (including time-dependent drift fields) follows for instance from the results in Landim, Olla, Yau [12] via the method of "the environment viewed from the particle". We use the pointwise 
Gaussian controls on the quenched semigroup from Norris [14] (see also Osada [19] for estimates that only accommodate a drift term with vanishing $\mathbb{P}$-expectation), that enable us to check condition $(T)$ by a standard Markov-process-type computation, see Theorem 3.1. With the help of our results in [21], we thus rederive the ballistic behavior (in the time-independent setting) of this class of diffusions in random environment. Let us also mention that, in the case where the $\mathbb{P}$-expectation of the drift is zero, diffusive behavior has been shown, see for instance [4],[15], [18] for the time-independent setting, and [3], [9], [12] for the time-dependent setting.

In Section 4, we consider Brownian motion perturbed by a random drift that can be written as the gradient of a non-stationary scalar potential. The ballistic behavior of this class of diffusions was only recently shown in Shen [22]. Whereas the method of "the environment viewed from the particle" applies successfully when the potential is stationary, see [16] or section 3.7 in [17], it does not apply in the above setting. Shen shows the existence of the first two moments of a certain regeneration time, see Theorem 4.11 in [22], and obtains the ballistic character as a direct consequence of his previous results, see Theorems 3.1 and 3.2. of [22]. With closely related techniques, we are able to prove in Theorem 4.1 that condition $(T)$ holds in this setting.

Let us also mention that in the context of the examples handled in Sections 3 and 4, the analogue of (8), where the annealed measure $P_{0}$ is replaced by the quenched measure $P_{0, \omega}$, holds uniformly in $\omega$, cf. (63) and (76).

Convention on constants: Unless otherwise stated, constants only depend on the quantities $\beta, \nu, K, R, d$. Dependence on additional parameters appears in the notation. Generic positive constants are denoted by $c$. When constants are not numerated, their value may change from line to line.

Acknowledgement: I am sincerely indebted to my advisor Prof. A.-S. Sznitman for his constant advice during the completion of this work. I would like to thank as well Laurent Goergen for fruitful discussions, and the referee for helpful comments.

\section{A new class of examples where Condition $(T)$ holds}

We first introduce some additional notation. For $z \in \mathbb{R}$, we denote with $\lfloor z\rfloor \stackrel{\text { def }}{=} \sup \{k \in \mathbb{Z}: k \leq$ $z$ \} the rounded value of $z$. We denote with $|\cdot|$ the Euclidean norm, and with $|\cdot|_{\infty}$ the supremum norm. For $x \in \mathbb{R}^{d}, r>0$, we write $B_{r}(x)$ for the open Euclidean ball with radius $r$ and center $x$. We call an open connected subset of $\mathbb{R}^{d}$ a domain, and for a subset $A$ of $\mathbb{R}^{d}$, we write $A^{c}$ for its complement, $\bar{A}$ for its closure, and $\partial A$ for its boundary. The diameter of $A$ is defined as $\operatorname{diam} A=\sup \{d(x, y): x, y \in A\}$. For two sets $A, B$ in $\mathbb{R}^{d}, d(A, B)=\inf \{|x-y|: x \in A, y \in B\}$ stands for the distance between $A$ and $B$. For an open subset $U$ of $\mathbb{R}^{d}$, the $\left(\mathcal{F}_{t}\right)$-stopping time $T_{U}=\inf \left\{t \geq 0: X_{t} \notin U\right\}$ denotes the exit time from $U$.

Given measurable functions $a, b$ on $\mathbb{R}^{d}$ with values in the space of symmetric $d \times d$ matrices, resp. in $\mathbb{R}^{d}$, that satisfy the conditions in (3) with the respective constants $\nu, \beta$ (with $a(\cdot), b(\cdot)$ in place of $a(\cdot, \omega), b(\cdot, \omega)$ ), we say that the attached differential operator $\mathcal{L}$ is of class $\mathcal{N}(\nu, \beta)$.

Recall the convention on constants at the end of the introduction. The main result of this section is 
Theorem 2.1. $(d \geq 1)$

Assume (1)-(5). There is a constant $c_{1}>1$, such that for $\ell \in S^{d-1}$, the inequality

$$
\mathbb{E}\left[(b(0, \omega) \cdot \ell)_{+}\right]>c_{1} \mathbb{E}\left[(b(0, \omega) \cdot \ell)_{-}\right]
$$

implies $(T) \mid \ell$.

This is an extension of Theorem 5.2 in our previous work [21], where we proved the same statement under the additional assumption that $a \equiv I d$.

Remark 2.2. One can easily argue that $c_{1} \geq 1$, and the proof of Theorem 2.1 shows in fact that $c_{1}>1$. The true value of the constant is unknown. However, when $\mathbb{P}$-a.s., $(b(0, \omega) \cdot \ell)_{-}=0$, then Theorem 2.1 immediately implies, regardless of the value of $c_{1}$ :

$$
\begin{aligned}
& \text { Condition }(T) \text { holds when } d \geq 1 \text { and there is } \ell \in S^{d-1} \text { and } \delta>0, \\
& \text { such that } b(0, \omega) \cdot \ell \geq 0 \text { for all } \omega \in \Omega \text {, and } p_{\delta}=\mathbb{P}[b(0, \omega) \cdot \ell \geq \delta]>0 .
\end{aligned}
$$

If there is $\delta>0$ such that $p_{\delta}=1$, this is in the spirit of the non-nestling case, which is in fact already covered by Proposition 5.1 in [21], and else, of the marginal nestling case in the discrete setting, see Sznitman [26]. Of course, Theorem 2.1 also comprises more involved examples of condition $(T)$ where $b(0, \omega) \cdot \ell$ takes both positive and negative values for every $\ell \in S^{d-1}$. They correspond to the plain-nestling case in [26]. When $d \geq 2$, and when the covariance matrix $a$ is not the identity, the examples of condition $(T)$ corresponding to the marginal- resp. plainnestling case provide new examples of ballistic diffusions in random environment.

The main step in the proof of Theorem 2.1 is to construct for each bounded $C^{\infty}$-domain $U$ containing 0 an auxiliary diffusion with characteristics independent of the environment, that, when started at 0 , exhibits the same exit distribution from $U$ as the annealed diffusion in random environment started at 0 . We provide this key result in subsection 2.1, cf. Proposition 2.4.

In subsection 2.2, we prove Theorem 2.1 using slight variations on the methods from [21]. The auxiliary diffusion enables us to restore some Markovian character to the task of checking condition $(T)$. First, we show that condition $(T)$ is implied by a certain condition $(K)$, which is similar to Kalikow's condition in the discrete set-up. We then check condition $(K)$, which finishes the proof of Theorem 2.1.

\subsection{An auxiliary diffusion and its exit distribution}

Unless otherwise stated, $U$ denotes from now on a bounded $C^{\infty}$-domain containing 0 . Recall the subtransition density $p_{\omega, U}(s, x, \cdot)$ at time $s>0$ for the quenched diffusion started at $x \in \mathbb{R}^{d}$, that fulfills for each open set $G \subset U, P_{x, \omega}\left[X_{s} \in G, T_{U}>s\right]=\int_{G} p_{\omega, U}(s, x, y) d y$. It can for instance be defined via Duhamel's formula, see [24] p.331:

$$
p_{\omega, U}(s, x, y)=p_{\omega}(s, x, y)-E_{x, \omega}\left[p_{\omega}\left(s-T_{U}, X_{T_{U}}, y\right), T_{U}<s\right], \quad s>0, x, y \in \mathbb{R}^{d},
$$

where $p_{\omega}(s, x, \cdot)$ denotes the transition density corresponding to the quenched diffusion started at $x \in \mathbb{R}^{d}$. One then obtains the Green function for the quenched diffusion started at 0 and killed when exiting $U$ via

$$
g_{\omega, U}(x)=\int_{0}^{\infty} p_{\omega, U}(s, 0, x) d s, \quad x \in \mathbb{R}^{d} .
$$


We define the auxiliary diffusion and drift coefficients, see also (11), through

$$
\begin{array}{ll}
a_{U}^{\prime}(x)=\frac{\mathbb{E}\left[g_{\omega, U}(x) a(x, \omega)\right]}{\mathbb{E}\left[g_{\omega, U}(x)\right]}, \text { if } x \in U \backslash\{0\}, & a_{U}^{\prime}(x)=I d, \text { else, } \\
b_{U}^{\prime}(x)=\frac{\mathbb{E}\left[g_{\omega, U}(x) b(x, \omega)\right]}{\mathbb{E}\left[g_{\omega, U}(x)\right]}, \text { if } x \in U \backslash\{0\}, \quad b_{U}^{\prime}(x)=0, \quad \text { else, }
\end{array}
$$

and we denote with $\mathcal{L}^{\prime}$ the attached differential operator.

Notice that the definition of $a_{U}^{\prime}, b_{U}^{\prime}$ in 0 and outside $U$ is quite arbitrary. Since we are interested in the exit distribution from $U$ of a suitable diffusion started at 0 and attached to $a_{U}^{\prime}, b_{U}^{\prime}$, cf. Proposition 2.4 below, we only require that $a_{U}^{\prime}, b_{U}^{\prime}$ are uniformly elliptic and bounded outside $U$. Let us further mention that changing the value of $a_{U}^{\prime}(0)$ within the class of symmetric and elliptic matrices does not affect issues such as existence and uniqueness of the solution to the martingale problem for $\mathcal{L}^{\prime}$, see for instance [1] p. 149 and Theorem 1.2 p.132 therein.

The next lemma will be useful in the remainder of this section.

Lemma 2.3. $\mathcal{L}^{\prime} \in \mathcal{N}(\nu, \beta)$. Moreover, $a_{U}^{\prime}, b_{U}^{\prime}$, and $g_{\omega, U}$ are continuous at $U \backslash\{0\}$.

Proof. It follows from (3) and from the definition of $a_{U}^{\prime}, b_{U}^{\prime}$ that $\mathcal{L}^{\prime} \in \mathcal{N}(\nu, \beta)$. Theorem 4.5 p.141 in [5] states that the transition density $p_{\omega}(s, x, y)$ is jointly continuous in $s>0, x, y \in \mathbb{R}^{d}$. By Duhamel's formula, see (14), and dominated convergence, it follows that the subtransition $p_{\omega, U}(s, x, y)$ is continuous in $y \in U$. From Lemma 5.2 in the Appendix, and from similar computations as carried out between (83) and (85) in the Appendix, and applying dominated convergence, we obtain the continuity of $g_{\omega, U}$ in $U \backslash\{0\}$. Further, by the upper bound in Corollary 5.3 and dominated convergence, and by continuity of $a(\cdot, \omega)$ resp. $b(\cdot, \omega)$, see (4), we see that $a_{U}^{\prime}$ resp. $b_{U}^{\prime}$ are continuous at $U \backslash\{0\}$.

The following proposition restores some Markovian character to the exit problem of the diffusion $X$. under the annealed measure $P_{0}\left(\right.$ recall $P_{0}$ in (7)).

Proposition 2.4. $(d \geq 1)$ Let $U$ be a bounded $C^{\infty}$-domain containing 0 . Then there is a solution $P_{0, U}^{\prime}$ to the martingale problem for $\mathcal{L}^{\prime}$ started at 0 , such that $X_{T_{U}}$ has same law under $P_{0, U}^{\prime}$ and the annealed measure $P_{0}$.

In the sequel we will denote with $P_{0, U}^{\prime}$ any such measure and refer to it as an auxiliary measure, and we will denote with $E_{0, U}^{\prime}$ the corresponding expectation.

Remark 2.5. When $d \leq 2$, Exercises 7.3.3 and 7.3.4 p.192 in [23], and the fact that $\mathcal{L}^{\prime} \in \mathcal{N}(\nu, \beta)$, show that the martingale problem for $\mathcal{L}^{\prime}$ is well-posed, so that the auxiliary measure $P_{0, U}^{\prime}$ corresponds to the unique solution to the martingale problem. In higher dimensions, uniqueness to the martingale problem may fail, see Nadirashvili [13]. Uniqueness holds for instance when the set of discontinuities of the diffusion coefficient contains only a finite number of cluster points, see Krylov [11]. In our case, where the diffusion coefficient $a_{U}^{\prime}$ is in general discontinuous at the boundary $\partial U$, the question of uniqueness is open. However, since $a_{U}^{\prime}$ is continuous at $U \backslash\{0\}$, one can in fact show with the above result from Krylov [11] that two arbitrary solutions to the martingale problem for $\mathcal{L}^{\prime}$ started at 0 agree on $\mathcal{F}_{T_{U}}$, although we will not prove it here. As a consequence, every solution to the martingale problem for $\mathcal{L}^{\prime}$ started at 0 can be used as auxiliary measure, but we will not use this here. 


\subsubsection{The case $d=1$}

Proof of Proposition 2.4 when $d=1$. According to Remark 2.5, we denote with $P_{0, U}^{\prime}$ the unique solution to the martingale problem for $\mathcal{L}^{\prime}$ started at 0 . Corollary 4.8 p.317 in [8], combined with Remark 4.3 p.173 therein, shows the existence of a Brownian motion $W$ defined on $\left(C\left(\mathbb{R}_{+}, \mathbb{R}\right), \mathcal{F}, P_{0, U}^{\prime}\right)$ such that

$$
P_{0, U}^{\prime}-\text { a.s., } \quad X_{t}=\int_{0}^{t} \sqrt{a_{U}^{\prime}\left(X_{s}\right)} d W_{s}+\int_{0}^{t} b_{U}^{\prime}\left(X_{s}\right) d s
$$

Say that $U=\left(\alpha_{1}, \alpha_{2}\right), \alpha_{1}<0<\alpha_{2}$, and define a scale function $s$,

$$
s(x)=\int_{\alpha_{1}}^{x} \exp \left\{-2 \int_{\alpha_{1}}^{y} \frac{b_{U}^{\prime}(z)}{a_{U}^{\prime}(z)} d z\right\} d y, \quad x \in \mathbb{R} .
$$

Notice that $s \in C^{1}(\mathbb{R})$, and that, due to Lemma 2.3, the second derivative of $s$ exists outside $A=\left\{\alpha_{1}, 0, \alpha_{2}\right\}$, and satisfies

$$
\partial_{x}^{2} s(x)=-\frac{2 b_{U}^{\prime}(x)}{a_{U}^{\prime}(x)} \partial_{x} s(x), \quad x \in A^{c}, \quad \text { and hence, } \quad \mathcal{L}^{\prime} s=0 \text { on } A^{c} .
$$

On $A$, we define $\partial_{x}^{2} s$ through the equality in (19), so that $\mathcal{L}^{\prime} s=0$ on $\mathbb{R}$. Applying the generalised Ito rule, cf. problem 7.3 p.219 in [8], and taking expectations, we find that,

$$
E_{0, U}^{\prime}\left[s\left(X_{t \wedge T_{U}}\right)\right]=s(0)+E_{0, U}^{\prime}\left[\int_{0}^{t \wedge T_{U}} \mathcal{L}^{\prime} s\left(X_{u}\right) d u\right]=s(0), \quad t \geq 0
$$

Using $E_{0, U}^{\prime}\left[T_{U}\right]<\infty$, together with dominated convergence, we obtain

$$
E_{0, U}^{\prime}\left[s\left(X_{T_{U}}\right)\right]=s(0)
$$

A similar equation as (17) holds with $P_{0, \omega}$ in place of $P_{0, U}^{\prime}$, and $a(\cdot, \omega), b(\cdot, \omega)$ in place of $a_{U}^{\prime}(\cdot), b_{U}^{\prime}(\cdot)$ respectively. We proceed similarly as in (20) and below, and obtain from the definition of the Green function $g_{\omega, U}$, cf. (15),

$$
E_{0, \omega}\left[s\left(X_{T_{U}}\right)\right]=s(0)+E_{0, \omega}\left[\int_{0}^{T_{U}} \mathcal{L}_{\omega} s\left(X_{u}\right) d u\right] \stackrel{(15)}{=} \int_{\alpha_{1}}^{\alpha_{2}} g_{\omega, U}(x) \mathcal{L}_{\omega} s(x) d x
$$

We integrate out (22) with respect to $\mathbb{P}$, and find from the definition of $a_{U}^{\prime}, b_{U}^{\prime}$, see (16),

$$
E_{0}\left[s\left(X_{T_{U}}\right)\right]=s(0)+\int_{\alpha_{1}}^{\alpha_{2}} \mathbb{E}\left[g_{\omega, U}(x)\right] \mathcal{L}^{\prime} s(x) d x=s(0)
$$

Since $s\left(\alpha_{1}\right)=0,(21)$ and (23) imply that $P_{0, U}^{\prime}\left[X_{T_{U}}=\alpha_{2}\right]=P_{0}\left[X_{T_{U}}=\alpha_{2}\right]$, which is our claim. 


\subsubsection{The case $d \geq 2$}

The higher-dimensional case is more intricate. In Remark 2.6, we show that when $d \geq 3$, then $a_{U}^{\prime}$ is in general discontinuous at the origin. In Remark 2.7, we first recall from [21] the main points of the proof of Proposition 2.4 when in addition $a=I d$ holds. Then we explain that these methods fail when $a_{U}^{\prime}$ is discontinuous. Hence, in the setting of a general covariance matrix $a$, where $a_{U}^{\prime}$ is in general discontinuous, the arguments from [21] break down.

Remark 2.6. Theorem 4.1 p.80 in [2] states that there is a constant $c>0$, such that for all $\omega \in \Omega, \varepsilon>0$, there is $\eta>0$ such that if $x \in U,|x| \leq \eta$, then

$$
1-\varepsilon \leq \frac{g_{\omega, U}(x)}{c h_{\omega}(x)} \leq 1+\varepsilon,
$$

where $h_{\omega}(x)=\left(x^{t} \cdot a(0, \omega) x\right)^{\frac{2-d}{2}}$. Choose a real-valued sequence $\left\{r_{n}\right\}_{n}$ converging to 0 , and define sequences $x_{n}^{(i)}=r_{n} e_{i}$, where $e_{i}$ is the $i$-th unit vector of the canonical basis. We find with the help of (24) and dominated convergence that

$$
\lim _{n} a_{U}^{\prime}\left(x_{n}^{(i)}\right)=\mathbb{E}\left[a_{i i}(0, \omega)^{\frac{2-d}{2}} a(0, \omega)\right] / \mathbb{E}\left[a_{i i}(0, \omega)^{\frac{2-d}{2}}\right],
$$

so that the limit depends on $i \in\{1, \ldots, d\}$. Hence $a_{U}^{\prime}$ is in general discontinuous at 0 .

Let us point out that there are examples of diffusions, attached to uniformly elliptic, but discontinuous diffusion coefficients, that return to their starting point with probability one, cf. Proposition 3.1 p.104 in [1].

Remark 2.7. 1. Let us recall that in [21], the additional assumption that $a \equiv I d$ in Theorem 5.2 allowed us to choose for each bounded $C^{\infty}$-domain $U$ containing 0 a Brownian motion perturbed by a bounded measurable drift $b_{U}^{\prime}$ (defined as in (16)) as the (uniquely defined) auxiliary diffusion. We emphasize that in this setting $a_{U}^{\prime}=I d$.

We recall the main points in the proof of Proposition 5.4 in [21] (which corresponds to Proposition 2.4 when $a=I d$ ): from the martingale problem, and by definition of $b_{U}^{\prime}$, we derive a sort of "forward" equation, namely for all $f \in C^{2}(\bar{U})$,

$$
E_{0}\left[f\left(X_{T_{U}}\right)\right]-E_{0, U}^{\prime}\left[f\left(X_{T_{U}}\right)\right]=\int_{U}\left(\mathbb{E}\left[g_{\omega, U}(x)\right]-g_{U}^{\prime}(x)\right)\left(\frac{1}{2} \Delta+b_{U}^{\prime}(x) \nabla\right) f(x) d x,
$$

where $g_{\omega, U}$ and $g_{U}^{\prime}$ are the respective Green functions corresponding to the quenched resp. auxiliary diffusion started at 0 and killed when exiting $U$. We choose an arbitrary smooth function $\phi$, and we denote with $u$ the unique strong solution in the Sobolev space $W^{2, p}(U)$, $p>d$, to the problem

$$
1 / 2 \Delta u+b_{U}^{\prime} \nabla u=0 \text { in } U, \quad u=\phi \text { on } \partial U .
$$

By considering the perturbed Dirichlet problems $1 / 2 \Delta u_{n}+b_{U, n}^{\prime} \nabla u_{n}=0$ in $U, u_{n}=\phi$ on $\partial U$, where the $b_{U, n}^{\prime}$ are smooth and converge a.e. in $U$ to the bounded measurable drift $b_{U}^{\prime}$, we obtain smooth solutions $u_{n}$ that converge in $W^{2, p}(U)$ to $u$. Sobolev's inequality yields an upper bound, uniform in $n \geq 1$, of the supremum norm on $U$ of the gradients of the $u_{n}$. Since on $U, 1 / 2 \Delta u_{n}+b_{U}^{\prime} \nabla u_{n}=\left(b_{U}^{\prime}-b_{U, n}^{\prime}\right) \nabla u_{n}$ holds, we then conclude the proof by inserting the smooth functions $u_{n}$ in (25) and using dominated convergence. 
2. Unlike the above setting (where $a_{U}^{\prime}=I d$ ), $a_{U}^{\prime}$ is now in general discontinuous at the origin when $d \geq 3$, see Remark 2.6. However, the existence of a unique strong solution in the Sobolev space $W^{2, p}(U), p \geq 1$, for the Dirichlet problem corresponding to (26),

$$
\mathcal{L}^{\prime} u=0 \text { in } U, \quad u=\phi \text { on } \partial U
$$

is only guaranteed when $a_{U}^{\prime}$ is continuous on $\bar{U}$, see [6] p.241. Hence, we have no controls on derivatives as above, and the arguments from [21] break down.

To avoid controls on derivatives, we use a result of Krylov [11]. The specific choice of the auxiliary coefficients $a_{U}^{\prime}, b_{U}^{\prime}$ enables us to find a diffusion started at 0 and attached to $a_{U}^{\prime}, b_{U}^{\prime}$ that, killed when exiting $U$, admits $\mathbb{E}\left[g_{\omega, U}\right]$ as occupation time density in $U$, and we show that this diffusion exhibits the same exit distribution from $U$ as the annealed diffusion in random environment.

Proof of Proposition 2.4 when $d \geq 2$. From the martingale problem, we find for $f \in C^{2}(\bar{U})$ :

$$
E_{0, \omega}\left[f\left(X_{t \wedge T_{U}}\right)\right]=f(0)+E_{0, \omega}\left[\int_{0}^{t \wedge T_{U}} \mathcal{L}_{\omega} f\left(X_{s}\right) d s\right] .
$$

Using ellipticity and standard martingale controls, it is immediate that $\sup _{\omega} E_{0, \omega}\left[T_{U}\right]<\infty$. By dominated convergence, and by the definition of the quenched Green function $g_{\omega, U}$, cf. (15), we find that

$$
E_{0, \omega}\left[f\left(X_{T_{U}}\right)\right]=f(0)+\int_{U} g_{\omega, U}(y) \mathcal{L}_{\omega} f(y) d y .
$$

After $\mathbb{P}$-integration, and from the definition of $\mathcal{L}^{\prime}$, cf. $(16)$, we obtain that

$$
E_{0}\left[f\left(X_{T_{U}}\right)\right]=f(0)+\int_{U} \mathbb{E}\left[g_{\omega, U}(y)\right] \mathcal{L}^{\prime} f(y) d y
$$

In particular the assumption of Theorem 2.14 in [11] is satisfied (with the respective choice of the domains $Q=\mathbb{R}^{d}$ and $D=U$ in the notations of [11]), and we infer the existence of a process $X$ and a Brownian motion $W$, defined on some probability space $\left(C, \mathcal{C}, Q_{0}\right)$, such that

$$
Q_{0}-\text { a.s., } \quad X_{t}=\int_{0}^{t} \sigma_{U}^{\prime}\left(X_{s}\right) d W_{s}+\int_{0}^{t} b_{U}^{\prime}\left(X_{s}\right) d s
$$

where $\sigma_{U}^{\prime}$ is a measurable square root of $a_{U}^{\prime}$, i.e. $a_{U}^{\prime}=\sigma_{U}^{\prime} \sigma_{U}^{\prime t}$, and such that for every bounded measurable function $\psi$,

$$
\int_{U} \mathbb{E}\left[g_{\omega, U}(y)\right] \psi(y) d y=E_{0, U}^{\prime}\left[\int_{0}^{T_{U}} \psi\left(X_{s}\right) d s\right]
$$

where $E_{0, U}^{\prime}$ denotes the expectation with respect to the measure $P_{0, U}^{\prime}$ induced by $X$ on $\left(C\left(\mathbb{R}_{+}, \mathbb{R}^{d}\right), \mathcal{F}\right)$. Corollary 4.8 p.317 in [8] shows that $P_{0, U}^{\prime}$ solves the martingale problem for $\mathcal{L}^{\prime}$ started at 0 . Insert $\psi=1$ in (32) to find $E_{0, U}^{\prime}\left[T_{U}\right]=E_{0}\left[T_{U}\right]<\infty$, see below (28). For all 
$f \in C^{2}(\bar{U})$, we derive a similar equation as (28) under the measure $P_{0, U}^{\prime}$, so that, when letting $t \rightarrow \infty$, and using dominated convergence, we find that

$$
\begin{aligned}
E_{0, U}^{\prime}\left[f\left(X_{T_{U}}\right)\right]=f(0)+E_{0, U}^{\prime}\left[\int_{0}^{T_{U}} \mathcal{L}^{\prime} f\left(X_{s}\right) d s\right] & \stackrel{(32)}{=} f(0)+\int_{U} \mathbb{E}\left[g_{\omega, U}(y)\right] \mathcal{L}^{\prime} f(y) d y \stackrel{(30)}{=} E_{0}\left[f\left(X_{T_{U}}\right)\right] .
\end{aligned}
$$

Since $f \in C^{2}(\bar{U})$ is arbitrary, the claim of the proposition follows.

Remark 2.8. Although we will not use it here, one can show that in the terminology of Krylov [10], [11], $\mathbb{E}\left[g_{\omega, U}\right]$ is the unique (up to Lebesgue equivalence) Green function of $\mathcal{L}^{\prime}$ in $U$ with pole at 0 . (This is a rather straightforward consequence of (32) and the fact that any two solutions to the martingale problem for $\mathcal{L}^{\prime}$ started at 0 agree on $\mathcal{F}_{T_{U}}$, cf. Remark 2.5).

\subsection{The proof of Theorem 2.1}

We now recall from [21] the definition of condition $(K)$, which is similar to Kalikow's condition in the discrete set-up. With the help of Proposition 2.4, we show that it implies condition $(T)$. The proof of Theorem 2.1 is finally concluded by checking condition $(K)$. The proofs in this subsection are adaptations from the corresponding proofs in section 5 in [21].

Definition 2.9. $(d \geq 1)$ Let $\ell \in S^{d-1}$. We say that condition $(K) \mid \ell$ holds, if there is an $\epsilon>0$, such that for all bounded domains $U$ containing 0

$$
\inf _{x \in U \backslash\{0\}, d(x, \partial U)>5 R} b_{U}^{\prime}(x) \cdot \ell>\epsilon,
$$

with the convention $\inf \varnothing=+\infty$.

As alluded to above, the next step is

Proposition 2.10. $(K)|\ell \Rightarrow(T)| \ell$.

Proof. The set of $\ell \in S^{d-1}$ for which (33) holds is open and hence our claim will follow if for such an $\ell$ we show that

$$
\limsup _{L \rightarrow \infty} L^{-1} \log P_{0}\left[X_{T_{U_{\ell, b, L}}} \cdot \ell<0\right]<0 .
$$

Denote with $\Pi_{\ell}(w) \stackrel{\text { def }}{=} w-(w \cdot \ell) \ell, w \in \mathbb{R}^{d}$, the projection on the orthogonal complement of $\ell$, and define

$$
V_{\ell, b, L} \stackrel{\text { def }}{=}\left\{x \in \mathbb{R}^{d}:-b L<x \cdot \ell<L,\left|\Pi_{\ell}(x)\right|<L^{2}\right\} .
$$

In view of Proposition 2.4, we choose bounded $C^{\infty}$-domains $\tilde{V}_{\ell, b, L}$ such that

$$
V_{\ell, b, L} \subset\left\{x \in \mathbb{R}^{d}:-b L<x \cdot \ell<L,\left|\Pi_{\ell}(x)\right|<L^{2}+5 R\right\} \subset \tilde{V}_{\ell, b, L} \subset U_{\ell, b, L} .
$$

(When $d=1, \Pi_{\ell}(w) \equiv 0$, and we simply have that $U_{\ell, b, L}=V_{\ell, b, L}=\tilde{V}_{\ell, b, L}$.) We denote with $P_{0, \tilde{V}_{\ell, b, L}}^{\prime}$ an auxiliary measure, i.e. $P_{0, \tilde{V}_{\ell, b, L}}^{\prime}$ solves the martingale problem for $\mathcal{L}^{\prime}$ started at 0 , and 
$X_{T_{\tilde{V}_{\ell, b, L}}}$ has same law under $P_{0, \tilde{V}_{\ell, b, L}}^{\prime}$ and $P_{0}$, cf. Proposition 2.4. To prove (34), it will suffice to prove that

$$
\limsup _{L \rightarrow \infty} L^{-1} \log P_{0, \tilde{V}_{\ell, b, L}}^{\prime}\left[X_{T_{V_{\ell, b, L}}} \cdot \ell<L\right]<0 .
$$

Indeed, once this is proved, it follows from (36) that

$$
\limsup _{L \rightarrow \infty} L^{-1} \log P_{0, \tilde{V}_{\ell, b, L}}^{\prime}\left[X_{T_{\tilde{V}_{\ell, b, L}}} \cdot \ell<L\right]<0 .
$$

Then, by construction of $P_{0, \tilde{V}_{\ell, b, L}}^{\prime}$, (38) holds with $P_{0, \tilde{V}_{\ell, b, L}}^{\prime}$ replaced by $P_{0}$, and, using (36) once more, (34) follows.

We now prove (37). By (36) and (33), we see that for $x \in V_{\ell, b, L}$,

$$
b_{\tilde{V}_{\ell, b, L}}^{\prime}(x) \cdot \ell \geq \begin{cases}\epsilon, & \text { if }-b L+5 R<x \cdot \ell<L-5 R \text { and } x \neq 0, \\ -\beta, & \text { else } .\end{cases}
$$

We thus consider the process $X_{t} \cdot \ell$. We introduce the function $u(\cdot)$ on $\mathbb{R}$, which is defined on $[-b L, L]$ through

$$
u(r) \stackrel{\text { def }}{=} \begin{cases}\alpha_{1} e^{\alpha_{2} \frac{\epsilon}{\nu}(b L-5 R)}\left(\alpha_{3}-e^{4 \nu \beta(r-(-b L+5 R))}\right), & \text { if } r \in[-b L,-b L+5 R], \\ e^{-\alpha_{2} \frac{\epsilon}{\nu} r}, & \text { if } r \in(-b L+5 R, L-5 R), \\ \alpha_{4} e^{-\alpha_{2} \frac{\epsilon}{\nu}(L-5 R)}\left(\alpha_{5}-e^{4 \nu \beta(r-(L-5 R))}\right), & \text { if } r \in[L-5 R, L],\end{cases}
$$

and which is extended boundedly and in a $C^{2}$ fashion outside $[-b L, L]$, and such that $u$ is twice differentiable in the points $-b L$ and $L$. The numbers $\alpha_{i}, 1 \leq i \leq 5$, are chosen positive and independent of $L$, via

$$
\alpha_{5}=1+e^{20 \nu \beta R}, \alpha_{4}=e^{-20 \nu \beta R}, \alpha_{2}=\min \left(1, \frac{4 \nu^{2} \beta}{\epsilon} e^{-20 \nu \beta R}\right), \alpha_{1}=\frac{\epsilon \alpha_{2}}{4 \nu^{2} \beta}, \alpha_{3}=1+\frac{4 \nu^{2} \beta}{\epsilon \alpha_{2}} .
$$

Then, on $[-b L, L], u$ is positive, continuous and decreasing. In addition, one has with the definition $j(r)=u^{\prime}\left(r_{+}\right)-u^{\prime}\left(r_{-}\right)$,

$$
j(-b L+5 R)=0 \text {, and } j(L-5 R) \leq 0 .
$$

On $\mathbb{R}^{d}$ we define the function $\tilde{u}(x)=u(x \cdot \ell)$, and for $\lambda$ real, we define on $\mathbb{R}_{+} \times \mathbb{R}$ the function $v_{\lambda}(t, r) \stackrel{\text { def }}{=} e^{\lambda t} u(r)$, and on $\mathbb{R}_{+} \times \mathbb{R}^{d}$ the function $\tilde{v}_{\lambda}(t, x) \stackrel{\text { def }}{=} v_{\lambda}(t, x \cdot \ell)=e^{\lambda t} \tilde{u}(x)$. We will now find $\lambda_{0}$ positive such that

$$
v_{\lambda_{0}}\left(t \wedge T_{V_{\ell, b, L}}, X_{t \wedge T_{V_{\ell, b, L}}} \cdot \ell\right) \text { is a positive supermartingale under } P_{0, \tilde{V}_{\ell, b, L}}^{\prime} .
$$

Corollary 4.8 p.317 in [8], combined with Remark 4.3 p.173 therein, shows the existence of a $d$-dimensional Brownian motion $W$ defined on $\left(C\left(\mathbb{R}_{+}, \mathbb{R}^{d}\right), \mathcal{F}, P_{0, \tilde{V}_{\ell, b, L}}^{\prime}\right)$, such that

$$
P_{0, \tilde{V}_{\ell, b, L}}^{\prime}-\text { a.s., } \quad Y_{t} \stackrel{\text { def }}{=} X_{t} \cdot \ell=\int_{0}^{t} \sigma_{\tilde{V}_{\ell, b, L}}^{\prime}\left(X_{s}\right) \cdot \ell d W_{s}+\int_{0}^{t} b_{\tilde{V}_{\ell, b, L}}^{\prime}\left(X_{s}\right) \cdot \ell d s,
$$


where $\sigma_{\tilde{V}_{\ell, b, L}}^{\prime}$ is a measurable square root of $a_{\tilde{V}_{\ell, b, L}}^{\prime}$, i.e. $a_{\tilde{V}_{\ell, b, L}}^{\prime}=\sigma_{\tilde{V}_{\ell, b, L}}^{\prime} \sigma_{\tilde{V}_{\ell, b, L}^{\prime}}^{\prime t}$. Writing $u$ as a linear combination of convex functions, we find from the generalised Itô rule, see [8] p.218, that

$$
P_{0, \tilde{V}_{\ell, b, L}}^{\prime}-\text { a.s., } \quad u\left(Y_{t}\right)=1+\int_{0}^{t} D^{-} u\left(Y_{s}\right) d Y_{s}+\int_{-\infty}^{\infty} \Lambda_{t}(a) \mu(d a),
$$

where $D^{-} u$ is the left-hand derivative of $u, \Lambda(a)$ is the local time of $Y$ in $a$, and $\mu$ is the second derivative measure, i.e. $\mu([a, b))=D^{-} u(b)-D^{-} u(a), a<b$ real. Notice that the first derivative of $u$ exists and is continuous outside $L-5 R$, and the second derivative of $u$ exists (in particular) outside the Lebesgue zero set $A=\{-b L+5 R, 0, L-5 R\}$. Hence we find by definition of the second derivative measure, and with the help of equation $(7.3)$ in $[8]$ that $P_{0, \tilde{V}_{\ell, b, L}}^{\prime}$-a.s.,

$$
\begin{aligned}
\int_{-\infty}^{\infty} \Lambda_{t}(a) \mu(d a) & =\int_{-\infty}^{\infty} \Lambda_{t}(a) \mathbf{1}_{A^{c}}(a) u^{\prime \prime}(a) d a+\Lambda_{t}(L-5 R) j(L-5 R) \\
& =\frac{1}{2} \int_{0}^{t} u^{\prime \prime}\left(Y_{s}\right) \mathbf{1}_{A^{c}}\left(Y_{s}\right) d\langle Y\rangle_{s}+\Lambda_{t}(L-5 R) j(L-5 R) .
\end{aligned}
$$

Another application of equation (7.3) p.218 in [8] shows that

$$
P_{0, \tilde{V}_{\ell, b, L}}^{\prime} \text { - a.s., } \quad \int_{0}^{t} \mathbf{1}_{A}\left(Y_{s}\right) d\langle Y\rangle_{s}=2 \int_{-\infty}^{\infty} \mathbf{1}_{A}(a) \Lambda_{t}(a) d a=0 .
$$

Since $d\langle Y\rangle_{s}=l \cdot a_{\tilde{V}_{\ell, b, L}}^{\prime}\left(X_{s}\right) l d s \geq d s / \nu$, see Lemma 2.3, we deduce from (46) that,

$$
P_{0, \tilde{V}_{\ell, b, L}}^{\prime} \text { - a.s., } \int_{0}^{t} \mathbf{1}_{A}\left(Y_{s}\right) d s=0 \text {, and hence, } P_{0, \tilde{V}_{\ell, b, L}}^{\prime}-\text { a.s., } \int_{0}^{t} D^{-} u\left(Y_{s}\right) \mathbf{1}_{A}\left(Y_{s}\right) d Y_{s}=0 .
$$

Combining (45) and (47), and by definition of the operator $\mathcal{L}^{\prime}$, see below (16), we can now rewrite (44) as the $P_{0, \tilde{V}_{\ell, b, L}}^{\prime}$-a.s. equalities

$$
\begin{aligned}
u\left(Y_{t}\right) & =1+\int_{0}^{t} u^{\prime}\left(Y_{s}\right) \mathbf{1}_{A^{c}}\left(Y_{s}\right) d Y_{s}+\frac{1}{2} \int_{0}^{t} u^{\prime \prime}\left(Y_{s}\right) \mathbf{1}_{A^{c}}\left(Y_{s}\right) d\langle Y\rangle_{s}+\Lambda_{t}(L-5) j(L-5 R) \\
& =1+\int_{0}^{t} \mathcal{L}^{\prime} \tilde{u}\left(X_{s}\right) \mathbf{1}_{A^{c}}\left(Y_{s}\right) d s+\Lambda_{t}(L-5 R) j(L-5 R)+M_{t},
\end{aligned}
$$

where $M_{t}$ is a continuous martingale. In particular, $\tilde{u}\left(X_{t}\right)\left(=u\left(Y_{t}\right)\right)$ is a continuous semimartingale, and applying Itô's rule to the product $e^{\lambda t} \cdot \tilde{u}\left(X_{t}\right)=\tilde{v}_{\lambda}\left(t, X_{t}\right)$, and using (47) once again, we obtain that, $P_{0, \tilde{V}_{\ell, b, L}}^{\prime}$-a.s.,

$$
\begin{aligned}
& v_{\lambda}\left(t, X_{t}\right)=1+\int_{0}^{t} \lambda e^{\lambda s} \tilde{u}\left(X_{s}\right) d s+\int_{0}^{t} e^{\lambda s} d \tilde{u}\left(X_{s}\right) \\
& =1+\int_{0}^{t}\left(\frac{\partial}{\partial s}+\mathcal{L}^{\prime}\right) \tilde{v}_{\lambda}\left(s, X_{s}\right) \mathbf{1}_{A^{c}}\left(Y_{s}\right) d s+j(L-5 R) \int_{0}^{t} e^{\lambda s} d \Lambda_{s}^{L-5 R}+N_{t},
\end{aligned}
$$

where $N_{t}$ is a continuous martingale. Using $\frac{1}{\nu} \leq l \cdot a_{\tilde{V}_{\ell, b, L}}^{\prime}(x) l \leq \nu$, we find through direct computation that for $x \in V_{\ell, b, L}$, and a suitable $\psi(x) \geq 0$, using the notation $I_{1}=(-b L,-b L+$ 
$5 R), I_{2}=(-b L+5 R, L-5 R), I_{3}=(L-5 R, L)$,

$$
\left[\left(\frac{\partial}{\partial s}+\mathcal{L}^{\prime}\right) \tilde{v}_{\lambda}\right](s, x) \leq \psi(x) e^{\lambda s} \cdot \begin{cases}\lambda\left(e^{20 \nu \beta R} \alpha_{3}-1\right)-4 \nu \beta\left(2 \beta+b_{\tilde{V}_{\ell, b, L}}^{\prime}(x) \cdot \ell\right), & \text { if } x \cdot \ell \in I_{1}, \\ \lambda+\alpha_{2} \frac{\epsilon}{\nu}\left(\frac{1}{2} \alpha_{2} \epsilon-b_{\tilde{V}_{\ell, b, L}}^{\prime}(x) \cdot \ell\right), & \text { if } x \cdot \ell \in I_{2}, \\ \lambda\left(\alpha_{5}-1\right)-4 \nu \beta\left(2 \beta+b_{\tilde{V}_{\ell, b, L}}^{\prime}(x) \cdot \ell\right), & \text { if } x \cdot \ell \in I_{3} .\end{cases}
$$

Hence, by (39) and (41), we can find $\lambda_{0}>0$ small such that for $x \in V_{\ell, b, L}, x \cdot \ell \notin A$, the right-hand side of the last expression is negative. Since $j(L-5 R) \leq 0$, see $(42)$, we obtain from (48) applied to the stopping time $t \wedge T_{V_{\ell, b, L}}$ that (43) holds.

We now derive the claim of the proposition from (43). When $d \geq 2$, the probability to exit $V_{\ell, b, L}$ neither from the "right" nor from the "left" can be bounded as follows:

$$
\begin{aligned}
& P_{0, \tilde{V}_{\ell, b, L}}^{\prime}\left[-b L<X_{T_{V_{\ell, b, L}}} \cdot \ell<L\right] \leq \\
& P_{0, \tilde{V}_{\ell, b, L}}^{\prime}\left[-b L<X_{T_{V_{\ell, b, L}}} \cdot \ell<L, T_{V_{\ell, b, L}}>\frac{2 \alpha_{2} \epsilon}{\lambda_{0}} L\right]+P_{0, \tilde{V}_{\ell, b, L}}^{\prime}\left[\sup \left|X_{t}\right| \geq L^{2}: t \leq \frac{2 \alpha_{2} \epsilon}{\lambda_{0}} L\right] .
\end{aligned}
$$

By Chebychev's inequality and Fatou's lemma, we find that the first term on the right-hand side is smaller than

$$
\begin{aligned}
& \frac{1}{v_{\lambda_{0}}\left(\frac{2 \alpha_{2} \epsilon}{\lambda_{0}} L, L\right)} E_{0, \tilde{V}_{\ell, b, L}}^{\prime}\left[v_{\lambda_{0}}\left(T_{V_{\ell, b, L}}, X_{T_{V_{\ell, b, L}}} \cdot \ell\right)\right] \\
\leq & c(\epsilon) e^{-\alpha_{2} \epsilon L} \liminf _{t \rightarrow \infty} E_{0, \tilde{V}_{\ell, b, L}}^{\prime}\left[v_{\lambda_{0}}\left(t \wedge T_{V_{\ell, b, L}}, X_{t \wedge T_{V_{\ell, b, L}}} \cdot \ell\right)\right] \\
\leq & c(\epsilon) e^{-\alpha_{2} \epsilon L} v_{\lambda_{0}}(0,0)=c(\epsilon) e^{-\alpha_{2} \epsilon L},
\end{aligned}
$$

where, in the last inequality, we used (43). Applying Lemma 5.1 iii) to the second term in the right-hand side of (49), we obtain together with (50), that

$$
\limsup _{L \rightarrow \infty} L^{-1} \log P_{0, \tilde{V}_{\ell, b, L}}^{\prime}\left[-b L<X_{T_{V_{\ell, b, L}}} \cdot \ell<L\right]<0 .
$$

When $d \geq 1$, we bound the probability to exit $V_{\ell, b, L}$ from the left by a similar argument as in (50), and find that

$$
P_{0, \tilde{V}_{\ell, b, L}}^{\prime}\left[X_{T_{V_{\ell, b, L}}} \cdot \ell=-b L\right] \leq \frac{v_{\lambda_{0}}(0,0)}{v_{\lambda_{0}}(0,-b L)} \leq e^{-c(\epsilon) L} .
$$

(52), together with (51), when $d \geq 2$, show (37), which implies condition $(T) \mid \ell$.

Now the proof of Theorem 2.1 is carried out by checking condition $(K) \mid \ell$. The proof remains the same as for the case $a=I d$, which can be found in [21], see (5.38) and the subsequent computations.

This finishes the proof of Theorem 2.1.

\section{Condition $(T)$ for a diffusion with divergence-free drift}

In this section, we assume that $d \geq 2$. On $(\Omega, \mathcal{A}, \mathbb{P})$ we consider a random variable $H(\cdot)$ with values in the space of skew-symmetric $d \times d$-matrices, and we define for $\omega \in \Omega, x \in \mathbb{R}^{d}$, 
$H(x, \omega)=H\left(t_{x} \omega\right)$, so that $H$ is stationary under $\mathbb{P}$. We assume that $H$ obeys finite range dependence, i.e.

(5) holds with $H$ in place of $a, b$,

and that $H$ is bounded and has bounded and Lipschitz continuous derivatives, i.e. there are constants $\eta, \tilde{\beta}, \tilde{K}$ such that for all $1 \leq i, j, k \leq d, \omega \in \Omega$,

- $|H(\cdot, \omega)| \leq \eta$

- $\partial_{k} H_{i j}(\cdot, \omega)$ exists, $\left|\partial_{k} H_{i j}(\cdot, \omega)\right| \leq \tilde{\beta}$, and

- $\left|\partial_{k} H_{i j}(y, \omega)-\partial_{k} H_{i j}(z, \omega)\right| \leq \tilde{K}|y-z|$, for all $y, z \in \mathbb{R}^{d}$.

For a constant vector $v \neq 0$, we define diffusion and drift coefficients as

$$
a(\cdot, \omega)=I d, \quad b(\cdot, \omega)=\nabla \cdot H(\cdot, \omega)+v, \omega \in \Omega,
$$

i.e. for $1 \leq j \leq d, b_{j}(\cdot, \omega)=\sum_{i=1}^{d} \partial_{i} H_{i j}(\cdot, \omega)+v_{j}$. It follows from the skew-symmetry of $H$ and from (57) that for all $\omega, \nabla \cdot b(\cdot, \omega)=0$ in the weak sense. Then the measures $P_{x, \omega}$ can be viewed as describing the motion of a diffusing particle in an incompressible fluid and equipped with a random stationary velocity field obeying finite range dependence. It further follows from (57) that the operator $\mathcal{L}_{\omega}$ defined in (6) can be written with a principal part in divergence form:

$$
\mathcal{L}_{\omega}=\nabla \cdot\left(\left(\frac{1}{2} I d+H(\cdot, \omega)\right) \nabla\right)+v \nabla .
$$

Theorem 3.1. $(d \geq 2)$

Assume (54), (55) and (57). Then $(T) \mid \hat{v}$ holds, where $\hat{v}=\frac{v}{|v|}$. Moreover we have a strong law of large numbers with an explicit velocity:

$$
\text { for all } \omega \in \Omega, P_{0, \omega}-\text { a.s., } \quad \lim _{t \rightarrow \infty} \frac{X_{t}}{t} \longrightarrow \mathbb{E}[b(0, \omega)]=v .
$$

If (53)-(57) hold, then in addition a functional CLT holds:

$$
\text { under } P_{0}, B^{s}=\frac{X_{\cdot s}-\cdot s \mathbb{E}[b(0, \omega)]}{\sqrt{s}} \text { converges in law on } C\left(\mathbb{R}_{+}, \mathbb{R}^{d}\right) \text {, as } s \rightarrow \infty \text {, to a }
$$

Brownian motion B. with non-degenerate covariance matrix.

Proof. We recall the convention on constants stated at the end of the Introduction. It follows from (55)-(57) that our standing assumptions (3), (4) hold with $\nu=1, \beta=d^{2} \tilde{\beta}+d|v|$ and $K=d^{2} \tilde{K}$. The following upper bound on the heat kernel from Theorem 1.1 in Norris [14] will be instrumental in checking $(T) \mid \hat{v}$ and in proving (59): there is a constant $c_{2}$ that depends only on $\eta$ and $d$, such that for all $\omega \in \Omega, x, y \in \mathbb{R}^{d}$ and $t>0$,

$$
p_{\omega}(t, x, y+v t) \leq c_{2} t^{-d / 2} \exp \left\{-\frac{|y-x|^{2}}{c_{2} t}\right\} .
$$

We first prove (59). Choose $\varepsilon>0$. For $n \geq 1$, we define $A_{n}=\left\{\left|\frac{X_{n}}{n}-v\right|>\varepsilon\right\}$ and $B_{n}=\left\{\frac{Z_{1} \circ \theta_{n}}{n}>\right.$ $\varepsilon\}$ (recall $Z_{1}$ in Lemma 5.1). With the help of (61), we find for $\omega \in \Omega$, and for $n$ large,

$$
P_{0, \omega}\left[A_{n}\right]=\int_{B_{\varepsilon n}^{c}(0)} p_{\omega}(n, 0, y+n v) d y \leq \int_{B_{\varepsilon n}^{c}(0)} c_{2} n^{-d / 2} e^{-\frac{|y|^{2}}{c_{2} n}} d y \leq c_{2}^{2} n^{1-d / 2} e^{-\frac{\varepsilon^{2}}{2 c_{2}} n} .
$$


From the Markov property, and from Lemma 5.1 ii), we find that

$$
\sup _{\omega} P_{0, \omega}\left[B_{n}\right] \leq \sup _{x, \omega} P_{x, \omega}\left[Z_{1}>\varepsilon n\right] \leq \tilde{c}(\varepsilon) e^{-c(\varepsilon) n^{2}} .
$$

For $t>0$, we write

$$
\frac{X_{t}}{t}=\frac{\lfloor t\rfloor}{t}\left(\frac{X_{\lfloor t\rfloor}}{\lfloor t\rfloor}+\frac{X_{t}-X_{\lfloor t\rfloor}}{\lfloor t\rfloor}\right)
$$

and an application of Borel-Cantelli's lemma to the sets $A_{n}$ resp. $B_{n}$ shows that for all $\omega \in \Omega$, $P_{0, \omega}$-a.s., $\lim _{t} \frac{X_{t}}{t}=v$. Observe that, due to the stationarity of $b$ and $H$, for all $x \in \mathbb{R}^{d}$,

$$
\mathbb{E}[b(0, \omega)]=\mathbb{E}[b(x, \omega)]=\nabla \cdot \int_{\Omega} H\left(t_{x} \omega\right) \mathbb{P}(d \omega)+v=\nabla \cdot \int_{\Omega} H(\omega) \mathbb{P}(d \omega)+v=v,
$$

and (59) follows. Once we have checked $(T) \mid \hat{v}$, then (60) immediately follows from (10) and (59). To conclude the proof of Theorem 3.1, it thus remains to show that $(T) \mid \hat{v}$ holds.

For $x \in \mathbb{R}^{d}$ and $\ell \in S^{d-1}$, define the projection on the orthogonal complement of $\ell$, as well as a bounded approximation $V_{\ell, b, L}$ of the slab $U_{\ell, b, L}$,

$$
\Pi_{\ell}(x) \stackrel{\text { def }}{=} x-(x \cdot \ell) \ell, \quad V_{\ell, b, L} \stackrel{\text { def }}{=}\left\{x \in U_{\ell, b, L}:\left|\Pi_{\ell}(x)\right|<L^{2}\right\} .
$$

We choose unit vectors $\ell^{\prime}$ such that $\hat{v} \cdot \ell^{\prime}>\frac{2}{3}$, and we will show that for all such $\ell^{\prime}$ and all $b>0$,

$$
\limsup _{L \rightarrow \infty} L^{-1} \sup _{\omega \in \Omega} \log P_{0, \omega}\left[X_{T_{V_{\ell^{\prime}, b, L}}} \cdot \ell^{\prime}<L\right]<0,
$$

which clearly implies condition $(T) \mid \hat{v}$. Define $\gamma=\frac{3}{|v|}$, and observe that for all $\omega \in \Omega$,

$$
P_{0, \omega}\left[X_{T_{V_{\ell^{\prime}, b, L}}} \cdot \ell^{\prime}<L\right] \leq P_{0, \omega}\left[X_{T_{V_{\ell^{\prime}, b, L}}} \cdot \ell^{\prime}<L, T_{V_{\ell^{\prime}, b, L}} \leq \gamma L\right]+P_{0, \omega}\left[T_{V_{\ell^{\prime}, b, L}}>\gamma L\right] .
$$

We first estimate the second term on the right-hand side. Notice that for all $y \in V_{\ell^{\prime}, b, L}, \mid y-$ $\gamma L v \mid \geq\left(\left(L \ell^{\prime}-\gamma L v\right) \cdot \ell^{\prime}\right)_{-}=L\left(3 \hat{v} \cdot \ell^{\prime}-1\right)>L$. An application of (61) yields that

$$
\begin{aligned}
& \sup _{\omega} P_{0, \omega}\left[T_{V_{\ell^{\prime}, b, L}}>\gamma L\right] \leq \sup _{\omega} P_{0, \omega}\left[X_{\gamma L} \in V_{\ell^{\prime}, b, L}\right] \\
& \leq c_{2} \gamma^{-d / 2} L^{-d / 2} \int_{V_{\ell^{\prime}, b, L}} \exp \left\{-\frac{|y-v \gamma L|^{2}}{c_{2} \gamma L}\right\} d y \leq c(b, \gamma, \eta) L^{3 d / 2-1} \exp \left\{-\frac{L}{c_{2} \gamma}\right\} .
\end{aligned}
$$

In view of (63) and (64), it remains to show that for all $b>0$, and $\ell^{\prime}$ as above (63),

$$
\limsup _{L \rightarrow \infty} L^{-1} \sup _{\omega \in \Omega} \log P_{0, \omega}\left[X_{T_{V_{\ell^{\prime}, b, L}}} \cdot \ell^{\prime}<L, T_{V_{\ell^{\prime}, b, L}} \leq \gamma L\right]<0 .
$$

Introduce the subsets of $V_{\ell^{\prime}, b, L}$,

$$
V_{\ell^{\prime}, b, L}^{-} \stackrel{\text { def }}{=}\left\{x \in V_{\ell^{\prime}, b, L}: x \cdot \ell^{\prime}<-\frac{b L}{2}\right\}, V_{\ell^{\prime}, b, L}^{0} \stackrel{\text { def }}{=}\left\{x \in V_{\ell^{\prime}, b, L}:\left|\Pi_{\ell^{\prime}}(x)\right|>\frac{L^{2}}{2}\right\},
$$

as well as the union of these two sets, $\tilde{V}_{\ell^{\prime}, b, L} \stackrel{\text { def }}{=} V_{\ell^{\prime}, b, L}^{-} \cup V_{\ell^{\prime}, b, L}^{0}$, and write

$$
\begin{aligned}
& P_{0, \omega}\left[X_{T_{V_{\ell^{\prime}, b, L}}} \cdot \ell^{\prime}<L, T_{V_{\ell^{\prime}, b, L}} \leq \gamma L\right] \leq P_{0, \omega}\left[T_{V_{\ell^{\prime}, b, L}} \leq 1\right]+ \\
+ & \sum_{n=1}^{\lfloor\gamma L\rfloor} P_{0, \omega}\left[T_{V_{\ell^{\prime}, b, L}} \in(n, n+1], X_{T_{V_{\ell^{\prime}, b, L}}} \cdot \ell^{\prime}<L, X_{n} \notin \tilde{V}_{\ell^{\prime}, b, L}\right]+\sum_{n=1}^{\lfloor\gamma L\rfloor} P_{0, \omega}\left[X_{n} \in \tilde{V}_{\ell^{\prime}, b, L}\right] .
\end{aligned}
$$


Lemma 5.1 ii) shows that $\sup _{\omega} P_{0, \omega}\left[T_{V_{\ell^{\prime}, b, L}} \leq 1\right] \leq \tilde{c}(b) \exp \left\{-c(b) L^{2}\right\}$. With the help of the Markov property and Lemma 5.1 ii) we find that for large $L$, the middle term on the right-hand side of (67) is smaller than

$$
\begin{aligned}
\sum_{n=1}^{\lfloor\gamma L\rfloor} \sup _{\omega} P_{0, \omega}\left[\sup _{0 \leq t \leq 1}\left|X_{t}-X_{0}\right| \circ \theta_{n}>\frac{b L}{2}\right] & \leq \gamma L \sup _{x, \omega} P_{x, \omega}\left[\sup _{0 \leq t \leq 1}\left|X_{t}-X_{0}\right|>\frac{b L}{2}\right] \\
& \leq \tilde{c}(b, \gamma) L \exp \left\{-c(b) L^{2}\right\} .
\end{aligned}
$$

We now bound the third term on the right-hand side of (67):

$$
\sum_{n=1}^{\lfloor\gamma L\rfloor} \sup _{\omega} P_{0, \omega}\left[X_{n} \in \tilde{V}_{\ell^{\prime}, b, L}\right] \leq \sum_{n=1}^{\lfloor\gamma L\rfloor} \sup _{\omega} P_{0, \omega}\left[X_{n} \in V_{\ell^{\prime}, b, L}^{-}\right]+\gamma L \sup _{\omega} P_{0, \omega}\left[\sup _{t \leq \gamma L}\left|X_{t}\right|>L^{2} / 2\right] .
$$

Lemma 5.1 iii) shows that the second term on the right-hand side is smaller than $\tilde{c}(\gamma) L \exp \left\{-c(\gamma) L^{3}\right\}$. Using (61) we can bound the first term on the right-hand side of $(69)$ with

$$
\sum_{n=1}^{\lfloor\gamma L\rfloor} \int_{V_{\ell^{\prime}, b, L}^{-}} c_{2} n^{-d / 2} \exp \left\{-\frac{|y-n v|^{2}}{c_{2} n}\right\} \mathrm{d} y \leq c(b, \gamma, \eta) L^{2 d} \exp \left\{-\frac{b^{2}}{4 c_{2} \gamma} L\right\},
$$

where, in the last inequality, we simply used that $1 \leq n \leq \gamma L$ and, and that for all $n \geq 1$, and $y \in V_{\ell^{\prime}, b, L}^{-},|y-n v| \geq\left((y-n v) \cdot \ell^{\prime}\right)_{-} \geq b L / 2$ since $\ell^{\prime} \cdot \hat{v}>0$. We now obtain (66) by collecting the results between (67) and (70).This finishes the proof of the theorem.

\section{Condition $(T)$ for an anisotropic gradient-type diffusion}

Let $\varphi$ be a real-valued random variable on $(\Omega, \mathcal{A}, \mathbb{P})$, and define for $\omega \in \Omega, x \in \mathbb{R}^{d}, \varphi(x, \omega) \stackrel{\text { def }}{=}$ $\varphi\left(t_{x} \omega\right)$, so that $\varphi$ is stationary. We assume that $\varphi$ obeys finite range dependence, i.e.

(5) holds with $\varphi$ in place of $a, b$,

and that $\varphi$ is bounded and has bounded and Lipschitz continuous derivatives, i.e. there are constants $\eta, \beta, K$ such that for all $1 \leq i, j, k \leq d, \omega \in \Omega$,

$$
\begin{aligned}
& -|\varphi(\cdot, \omega)| \leq \eta \\
& \text { - }|\nabla \varphi(\cdot, \omega)| \leq \tilde{\beta} \text {, and }|\nabla \varphi(y, \omega)-\nabla \varphi(z, \omega)| \leq K|y-z|, y, z \in \mathbb{R}^{d} .
\end{aligned}
$$

Fix $\lambda>0$ and $\ell \in S^{d-1}$, and define the non-stationary function $\psi(x, \omega)=\varphi(x, \omega)+\lambda \ell \cdot x, \omega \in \Omega$, $x \in \mathbb{R}^{d}$. We now define diffusion and drift coefficients

$$
a(\cdot, \omega)=I d, \quad b(\cdot, \omega)=\nabla \psi(\cdot, \omega)=\nabla \varphi(\cdot, \omega)+\lambda \ell, \quad \text { all } \omega \in \Omega .
$$

It follows from (72) that

$$
e^{-2 \eta} e^{2 \lambda \ell \cdot x} \leq e^{2 \psi(x, \omega)} \leq e^{2 \eta} e^{2 \lambda \ell \cdot x}, \quad x \in \mathbb{R}^{d}, \omega \in \Omega .
$$

Under the above assumptions, Shen has shown that a ballistic law of large numbers and an invariance principle holds under the annealed measure $P_{0}$, see Section 4 of [22]. Our aim is to verify that condition $(T) \mid \ell$ holds. 
Theorem 4.1. $(d \geq 1)$ Condition $(T) \mid \ell$ holds under the assumptions (72)-(74).

Proof. We recall the convention on constants stated at the end of the Introduction. It follows from (73),(74) that our standing assumptions (3), (4) hold with $\nu=1, \beta=\tilde{\beta}+\lambda$ and $K$. We first introduce some notation. Recall the projection $\Pi$ in (62), and define for $\ell^{\prime} \in S^{d-1}$ and $\delta>0, V_{\ell^{\prime}, \delta}=\left\{x \in U_{\ell^{\prime}, b, L}:\left|\Pi_{\ell^{\prime}}(x)\right|<L / \sqrt{\delta}\right\}$. In fact, we will show that there exists $0<\delta<1$ such that for all $\ell^{\prime} \in S^{d-1}$ with $\ell^{\prime} \cdot \ell>\sqrt{1-\delta^{2}}$,

$$
\limsup _{L \rightarrow \infty} L^{-1} \sup _{\omega} \log P_{0, \omega}\left[X_{T_{V_{\ell^{\prime}, \delta}}} \cdot \ell^{\prime}<L\right]<0,
$$

which easily implies condition $(T) \mid \ell$. The strategy of proof of (76) relies on the methods introduced in Section 4.1 in Shen [22]. We recall some important facts from there.

We denote with $P_{\omega}^{t} f(x)=E_{x, \omega}\left[f\left(X_{t}\right)\right], f$ bounded measurable, the quenched semi-group generated by the operator $\mathcal{L}_{\omega}$ defined in (6), and with $m_{\omega}(d x)=\exp (2 \psi(x, \omega)) d x$ the reversible measure to $P_{\omega}^{t}$. The following key estimate is contained in Proposition 4.1 of [22]: there is a positive constant $c_{3}(\eta, \lambda)$ such that

$$
\sup _{\omega, U}\left\|P_{\omega, U}^{t}\right\|_{m_{\omega}} \leq \exp \left\{-c_{3} t\right\}, t>0
$$

where $\left(P_{\omega, U}^{t} f\right)(x) \stackrel{\text { def }}{=} E_{x, \omega}\left[f\left(X_{t}\right), T_{U}>t\right], t>0, f \in L^{2}\left(m_{\omega}\right),\|\cdot\|_{m_{\omega}}$ denotes the operator norm in $L^{2}\left(m_{\omega}\right)$ and $U$ varies over the collection of non-empty open subsets of $\mathbb{R}^{d}$.

The claim (76), and hence Theorem 4.1, follow from the next two propositions:

Proposition 4.2. Let $0<\delta<1$. There is a positive constant $c_{4}(\delta, b, \eta)$ such that for $\ell^{\prime} \in S^{d-1}$ with $\ell^{\prime} \cdot \ell>\sqrt{1-\delta^{2}}$ and $L>0$,

$$
\sup _{\omega} P_{0, \omega}\left[T_{V_{\ell^{\prime}, \delta}} \geq \frac{3 \lambda}{c_{3}} L\right] \leq c_{4} e^{-\frac{\lambda}{2} L} .
$$

Proposition 4.3. There exist $0<\delta<1$ and positive constants $c_{5}(\delta, b, \eta, \lambda), c_{6}(b, \eta, \lambda)$ such that for all $\ell^{\prime} \in S^{d-1}$ with $\ell^{\prime} \cdot \ell>\sqrt{1-\delta^{2}}$ and $L>0$,

$$
\sup _{\omega} P_{0, \omega}\left[T_{V_{\ell^{\prime}, \delta}}<\frac{3 \lambda}{c_{3}} L, X_{T_{\ell_{\ell^{\prime}, \delta}}} \cdot \ell^{\prime}<L\right] \leq c_{5} e^{-c_{6} L} .
$$

The proofs are close to the proofs of Propositions 4.2 and 4.3 in Shen [22]. We indicate the main steps of the proofs in the Appendix.

\section{Appendix}

\subsection{Bernstein's Inequality}

The following Lemma follows in essence from Bernstein's inequality (see [20] page 153-154). A proof of an inequality similar to those below can be found in the appendix of [21]. Recall the definition of the class of operators $\mathcal{N}(\nu, \beta)$ at the beginning of Section 2. 
Lemma 5.1. Let $\mathcal{L} \in \mathcal{N}(\nu, \beta)$, denote with $Q_{x}$ an arbitrary solution to the martingale problem for $\mathcal{L}$ started at $x \in \mathbb{R}^{d}$, and set $Z_{t}=\sup _{s \leq t}\left|X_{s}-X_{0}\right|$. For every $\gamma>0$, there are positive constants $c, \tilde{c}$, depending only on $\gamma, \nu, \beta, d$ such that for $L>0$,

$$
\begin{aligned}
& \text { i) } \sup _{x} Q_{x}\left[Z_{\gamma L} \geq 2 \gamma \beta L\right] \leq \tilde{c} e^{-c L}, \quad \text { ii) } \sup _{x} Q_{x}\left[Z_{1} \geq \gamma L\right] \leq \tilde{c} e^{-c L^{2}}, \\
& \text { iii) } \sup _{x} Q_{x}\left[Z_{\gamma L} \geq L^{2}\right] \leq \tilde{c} e^{-c L^{3}} .
\end{aligned}
$$

\subsection{Bounds on the transition density and on the Green function}

Recall the convention on the constants stated at the end of the Introduction.

Lemma 5.2. Let $\mathcal{L}_{\omega}$ be as in (6), and let assumptions (3) and (4) be in force. Then the linear parabolic equation of second order $\frac{\partial u}{\partial t}=\mathcal{L}_{\omega} u$ has a unique fundamental solution $p_{\omega}(t, x, y)$, and there are positive constants $c, \tilde{c}$, such that for $\omega \in \Omega$, and $0<t \leq 1$,

$$
\left|p_{\omega}(t, x, y)\right|<\frac{\tilde{c}}{t^{d / 2}} \exp \left\{-\frac{c|x-y|^{2}}{t}\right\} .
$$

For the proof of (81) we refer the reader to [7]. The statement (4.16) therein corresponds to (81). Recall the Green function $g_{\omega, U}$ in (15). In Corollary 6.3 in [21], we obtained the following

Corollary 5.3. Assume (3) and (4), and let $U$ be a bounded $C^{\infty}$-domain. There are positive constants $\tilde{c}, c(U)$ such that for $x \in U$, and all $\omega \in \Omega$,

$$
g_{\omega, U}(x) \leq \begin{cases}\tilde{c}|x|^{2-d}+c, & \text { if } d \geq 3 \text { and } x \neq 0, \\ \tilde{c} \log \frac{\operatorname{diam} U}{|x|}+c, & \text { if } d=2 \text { and } x \neq 0 \\ c, & \text { if } d=1\end{cases}
$$

Proof. We repeat here the computations from [21] for the convenience of the reader. From the definition of $g_{\omega, U}$ and $p_{\omega, U}$, see (15) and (14), we obtain

$$
g_{\omega, U}(x)=\int_{0}^{\infty} p_{\omega, U}(t, 0, x) d t \leq \int_{0}^{1} p_{\omega}(t, 0, x) d t+\sum_{k=2}^{\infty} \int_{\frac{k}{2}}^{\frac{k+1}{2}} p_{\omega, U}(t, 0, x) d t .
$$

With the help of (81), we find positive constants $\tilde{c}, c$ such that $\int_{0}^{1} p_{\omega}(t, 0, x) d t$ is smaller than the right-hand side of (82), and hence, it suffices to show that for some constant $c(U)$,

$$
\sup _{\omega} \sum_{k=2}^{\infty} \int_{\frac{k}{2}}^{\frac{k+1}{2}} p_{\omega, U}(t, 0, x) d t \leq c<\infty .
$$

We obtain by a repeated use of the Chapman-Kolmogorov equation and by (81), that for $k \geq 2$,

$$
\begin{aligned}
& \int_{\frac{k}{2}}^{\frac{k+1}{2}} p_{\omega, U}(t, 0, x) d t \leq \int_{U} p_{\omega, U}\left(\frac{1}{2}, 0, v\right) d v \sup _{v \in U} \int_{\frac{k-1}{2}}^{\frac{k}{2}} p_{\omega, U}(t, v, x) d t \\
& \quad \stackrel{\text { induction }}{\leq}\left(\sup _{v \in U} P_{v, \omega}\left[T_{U}>\frac{1}{2}\right]\right)^{k-1} \sup _{v \in U} \int_{\frac{1}{2}}^{1} p_{\omega, U}(t, v, z) d t \leq c\left(\sup _{v \in U} P_{v, \omega}\left[T_{U}>\frac{1}{2}\right]\right)^{k-1} .
\end{aligned}
$$

The Support Theorem of Stroock-Varadhan, see [1] p.25, shows that $\sup _{\omega, v \in U} P_{v, \omega}\left[T_{U}>\frac{1}{2}\right] \leq 1-c(U)$ for some positive constant $c(U)$, and (84) follows. 


\subsection{The proof of propositions 4.2 and 4.3}

Proof of Proposition 4.2. For sake of notations, we write $V$ and $B$ for $V_{\ell^{\prime}, \delta}$ resp. $B_{\frac{L(1 \wedge b)}{2}}(0)$. The Markov property and Cauchy-Schwarz's inequality yield for $t>0$

$$
\begin{aligned}
P_{0, \omega}\left[T_{V}>t\right] & \leq E_{0, \omega}\left[P_{X_{1}, \omega}\left[T_{V}>t-1\right], X_{1} \in B\right]+P_{0, \omega}\left[X_{1} \notin B\right] \\
& \leq\left\|1_{B}(\cdot) p_{\omega}(1,0, \cdot) e^{-2 \psi(\cdot, \omega)}\right\|_{L^{2}\left(m_{\omega}\right)}\left\|P_{\omega, V}^{t-1}\right\|_{m_{\omega}}\left\|1_{V}\right\|_{L^{2}\left(m_{\omega}\right)}+P_{0, \omega}\left[X_{1} \notin B\right],
\end{aligned}
$$

where $p_{\omega}(s, x, y)$ denotes the transition density function under the quenched measure $P_{x, \omega}$. Lemma 5.1 ii) shows that $P_{0, \omega}\left[X_{1} \notin B\right] \leq c(b) \exp \left(-c(b) L^{2}\right)$. Further, using (75) and Lemma 5.2 , we obtain

$$
\left\|1_{B}(\cdot) p_{\omega}(1,0, \cdot) e^{-2 \psi(\cdot, \omega)}\right\|_{L^{2}\left(m_{\omega}\right)}^{2} \leq c e^{2 \eta} \int 1_{B}(y) e^{-2 \lambda \ell \cdot y} d y \leq c(\eta, b) L^{d} e^{\lambda(1 \wedge b) L} .
$$

Choose a unit vector $\tilde{\ell}$, orthogonal to $\ell^{\prime}$, and such that $\tilde{\ell}$ lies in the span of $\ell$ and $\ell^{\prime}$. Then $\ell \cdot \ell^{\prime}>\sqrt{1-\delta^{2}}$ implies that $\ell \cdot \tilde{\ell}<\delta$, and for $y \in V$, we find that $y \cdot \ell \leq(1+\sqrt{\delta}) L<2 L$. As a result, and using again (75) and Lemma 5.2,

$$
\left\|1_{V}\right\|_{L^{2}\left(m_{\omega}\right)}^{2} \leq e^{2 \eta} \int 1_{V}(y) e^{2 \lambda \ell \cdot y} d y \leq c(\delta, \eta) L^{d} \exp \{4 \lambda L\} .
$$

Jointly with (77), we find that $P_{0, \omega}\left[T_{V}>t\right] \leq c(\delta, \eta, b) L^{d} \exp \left(\frac{5}{2} \lambda L\right) \exp \left(-c_{3} t\right)$, and the claim follows from the choice of $t$.

Proof of Proposition 4.3. We write $V$ for $V_{\ell^{\prime}, \delta}, \ell^{\prime} \in S^{d-1}$, and $B$ for $B_{\frac{(1 \wedge b) L}{4}}(0)$, and we recall the projection $\Pi$ in (62). We define

$$
V^{0} \stackrel{\text { def }}{=}\left\{x \in V:\left|\Pi_{\ell^{\prime}}(x)\right|>\frac{1}{2 \sqrt{\delta}} L\right\}, \quad V^{-\stackrel{\text { def }}{=}}\left\{x \in V: x \cdot \ell^{\prime}<-\frac{3}{4}(1 \wedge b) L\right\} .
$$

We proceed in a similar fashion as in the proof of Proposition 3.1, see (67) and the following lines, and find with $\mu_{0}=3 \lambda / c_{3}$ that

$$
P_{0, \omega}\left[T_{V}<\mu_{0} L, X_{T_{V}} \cdot \ell^{\prime}<L\right] \leq \sum_{n=1}^{\left\lfloor\mu_{0} L\right\rfloor} P_{0, \omega}\left[X_{n} \in V^{-} \cup V^{0}\right]+c(\delta, b) \exp \{-c(\delta, b) L\} .
$$

The first term in the right-hand side can be bounded by

$$
\sum_{n=1}^{\left\lfloor\mu_{0} L\right\rfloor} P_{0, \omega}\left[X_{n} \in V^{0}\right]+\sum_{n=1}^{\left\lfloor\mu_{0} L\right\rfloor} P_{0, \omega}\left[X_{n} \in V^{-}, X_{1} \in B\right]+\mu_{0} L P_{0, \omega}\left[X_{1} \notin B\right] .
$$

Lemma 5.1 ii) shows that the righmost term in (87) is smaller than $c(b, \eta, \lambda) L e^{-c(b) L}$, and, when in addition $\delta^{-1 / 2} \geq 4 \mu_{0} \beta$, Lemma 5.1 i) shows that the leftmost sum in (87) is smaller than $c(\eta, \lambda) L \exp \{-c(\eta, \lambda) L\}$. It thus suffices to bound the middle term in (87). Introduce the measure $m(d x)=\exp \{2 \lambda \ell \cdot x\} d x$. It follows from the Markov property and Lemma 5.2 that the second sum in (87) is smaller than

$$
\sum_{n=1}^{\left\lfloor\mu_{0} L\right\rfloor} \int_{B} p_{\omega}(1,0, y)\left(P_{\omega}^{n-1} 1_{V^{-}}\right)(y) d y \leq \sum_{n=1}^{\left\lfloor\mu_{0} L\right\rfloor} c \exp \left\{\frac{1}{2} \lambda(1 \wedge b) L\right\}\left\langle P_{\omega}^{n-1} 1_{V^{-}}, 1_{B}\right\rangle_{m}
$$


where, for two measurable functions $f, g,\langle f, g\rangle_{m}$ denotes the integral of the product $f \cdot g$ w.r.t. the measure $m$. It follows from Theorem 1.8 in [25] (for details see the proof of Proposition 4.3 in [22]) that there is a constant $c_{7}(\eta)$ such that for all $\omega \in \Omega$, and any open sets $A, D$ in $\mathbb{R}^{d}$,

$$
\left\langle P_{\omega}^{n-1} 1_{A}, 1_{D}\right\rangle_{m} \leq \sqrt{m(A)} \sqrt{m(D)} \exp \left\{-\frac{d(A, D)^{2}}{4 c_{7}(n-1)}\right\} .
$$

We find by definition of $B, V$ and, by a similar argument as given above (86), that

$$
m(B) \leq c(b) L^{d} \exp \left\{\frac{1}{2} \lambda(1 \wedge b) L\right\}, m\left(V^{-}\right) \leq c(\delta, b) L^{d} \exp \left\{-\frac{3}{2} \lambda(1 \wedge b) \sqrt{1-\delta^{2}} L+2 \lambda \sqrt{\delta} L\right\} .
$$

Since $d\left(B, V^{-}\right)=\frac{1}{2}(1 \wedge b) L,(89)$ applied to the open sets $B, V$, yields that for large $L$, the right-hand side of $(88)$ is smaller than

$$
\begin{aligned}
& \sum_{n=1}^{\left\lfloor\mu_{0} L\right\rfloor} c \exp \left\{\frac{1}{2} \lambda(1 \wedge b) L\right\} \sqrt{m(B)} \sqrt{m\left(V^{-}\right)} \exp \left\{-\frac{d\left(B, V_{-}\right)^{2}}{4 c_{7} \mu_{0} L}\right\} \\
\leq & c(\delta, b) \mu_{0} L^{d+1} \exp \{\sqrt{\delta} \lambda L\} \exp \left\{\frac{3}{4} \lambda(1 \wedge b)\left(1-\sqrt{1-\delta^{2}}\right) L\right\} \exp \left\{-\frac{(1 \wedge b)^{2}}{c(\lambda, \eta)} L\right\} \\
\leq & c(\delta, b) e^{-c(b, \eta, \lambda) L},
\end{aligned}
$$

provided $\delta$ is chosen small enough. This finishes the proof.

\section{References}

[1] Bass, R.: "Diffusions and Elliptic Operators", Springer Verlag, 1998.

[2] Boboc, N., Mustata, P.: "Espaces harmoniques associés aux opérateurs differentiels linéaires du second ordre de type elliptique", Lecture Notes in Math., volume 68, Springer Verlag, 1968.

[3] Fannjiang, A., Komorowski, T .: "An invariance principle for diffusion in turbulence ", Ann. Probab., 27(2), page 751-781, 1999.

[4] Fannjiang, A., Papanicolaou, G.: "Diffusion in turbulence", Probab. Theory relat. Fields, 105, page 279-334, 1996.

[5] Friedman, A.: "Stochastic Differential Equations and Applications, Vol.1, Academic Press, San Diego, 1975.

[6] Gilbarg, D., Trudinger, N.S.: "Elliptic Partial Differential Equations of the Second Order", Springer Verlag, 1998.

[7] Il'in, A.M., Kalashnikov, A.S., Oleinik, O.A.: "Linear equations of the second order of parabolic type", Russian Math. Surveys, 17(3), page 1-143, 1962.

[8] Karatzas, I., Shreve, S.: "Brownian Motion and Stochastic Calculus", Second Edition, Springer Verlag, 1991.

[9] Komorowski, T., Olla, S.: "On homogenization of time-dependent random flows", Probab. Theory relat. Fields, 121(1), page 98-116, 2001. 
[10] Krylov, N.V.: "On one-point weak uniqueness for elliptic equations", Comm. Partial Differential Equations, 17(11-12), page 1759-1784, 1992. MR1194740

[11] Krylov, N.V.: "On weak uniqueness for some diffusions with discontinuous coefficients", Stochastic Proc. Appl., 113(1), page 37-64, 2004. MR2078536

[12] Landim, C., Olla, S., Yau, H.T.: "Convection-diffusion equation with space-time ergodic random flow", Probab. Theory relat. Fields, 112, page 203-220, 1998.

[13] Nadirashvili, N.: "Nonuniqueness in the Martingale Problem and the Dirichlet Problem for Uniformly Elliptic Operators", Ann. Scuola Norm. Sup. Pisa Cl. Sci., 24(4), page 537-550, 1997.

[14] Norris, J.R.: "Long-time Behavior of Heat Flows", Archive for rational Mechanics and Analysis, 140(2), page 161-195, 1997.

[15] Oelschläger, K.: "Homogenization of a diffusion process in a divergence-free random field", Ann. Probab., 16(3), page 1084-1126, 1988.

[16] Olla, S.: "Homogenization of diffusion processes in random fields", Ecole Doctorale, Ecole Polytechnique, Palaiseau, 1994.

[17] Olla, S.: "Central limit theorems for tagged particles and for diffusions in random environment". In: "Milieux Aléatoires", Panoramas et Synthèses, Numéro 12, Société Mathématique de France, 2001.

[18] Osada, H.: "Homogenization of diffusion processes with random stationary coefficients", Lecture Notes in Math., volume 1021, page 507-517, Springer, Berlin, 1983.

[19] Osada, H.: "Diffusion processes with generators of generalized divergence form", J. Math. Kyoto Univ., 27(4), page 597-619, 1987. MR916761

[20] Revuz, D., Yor, M.: "Continuous Martingales and Brownian Motion", 3rd Edition, Springer Verlag, Berlin, 1999.

[21] Schmitz, T.: "Diffusions in random environment and ballistic behavior", accepted for publication in Ann. I. H. Poincaré PR, in press, available at http://dx.doi.org/, with doi:10.1016/j.anihpb.2005.08.003.

[22] Shen, L.: "On ballistic diffusions in random environment", Ann. I. H. Poincaré, PR 39(5), page 839-876, 2003.

[23] Stroock, D.W., Varadhan, S.R.S.: "Multidimensional Diffusion Processes", Springer Verlag, New York, 1979.

[24] Stroock, D.W.: "Diffusion semigroups corresponding to uniformly elliptic divergence form operators", in: Lecture Notes in Math., Vol. 1321, Springer Verlag, Berlin, page 316-347, 1988.

[25] Sturm, K.T.: "Analysis on local Dirichlet Spaces-II. Upper Gaussian estimates for the fundamental solutions of parabolic equations", Osaka J. Math., 32, page 275-312, 1995. 
[26] Sznitman, A.-S.: "Slowdown estimates and central limit theorem for random walks in random environment", J. Eur. Math. Soc., 2, page 93-143, 2000.

[27] Sznitman, A.-S.: "On a class of transient random walks in random environment", Ann. Probab., 29(2), page 723-764, 2001.

[28] Sznitman, A.-S.: "An effective criterion for ballistic behavior of random walks in random environment", Probab. Theory relat. Fields, 122(4), page 509-544, 2002.

[29] Sznitman, A.-S.: "On new examples of ballistic random walks in random environment", Ann. Probab., 31(1), page 285-322, 2003.

[30] Sznitman, A.-S.: "Topics in random walks in random environment", ICTP Lecture Notes Series, Volume XVII: School and Conference on Probability Theory, May 2004, available at www.ictp.trieste.it/ pub_off/lectures.

[31] Sznitman, A.-S., Zerner, M.P.W.: "A law of large numbers for random walks in random environment", Ann. Probab., 27(4), page 1851-1869, 1999.

[32] Sznitman, A.-S., Zeitouni, O.: "An Invariance Principle for Isotropic Diffusions in Random Environment", Invent. Math., 164(3), page 455-567, 2006. MR2221130

[33] Zeitouni, O.: "Random Walks in Random Environment", Lecture Notes in Mathematics, volume 1837, page 190-312, Springer, 2004. 